CONSTITUINTES QUÍMICOS E ATIVIDADES BIOLÓGICAS DE ÓLEOS ESSENCIAIS DAS ESPÉCIES Malva sylvestris e Pelargonium graveolens: UMA REVISÃO

\title{
CHEMICAL CONSTITUENTS AND BIOLOGICAL ACTIVITIES OF ESSENTIAL OILS OF THE SPECIES Malva sylvestris and Pelargonium graveolens: A REVIEW
}

\author{
Janine Machado Oliveira* \\ Universidade Federal do Sul e Sudeste do Pará, Marabá, PA, Brasil. \\ *janinemachaoliver@gmail.com
}

\section{RESUMO}

Determinadas plantas medicinais possuem nomes populares semelhantes entre si. Malva sylvestris e Pelargonium graveolens são espécies conhecidas pelo mesmo nome popular "Malva" e, portanto, comumente confundidas, além de serem usadas para fins medicinais parecidos. O objetivo do trabalho foi executar uma revisão da literatura dos últimos dez anos sobre o desenvolvimento do conhecimento científico de óleos essenciais de $M$. sylvestris e $P$. graveolens, com a finalidade de identificar os principais componentes químicos e atividades biológicas. Os resultados descrevem a produção comercial destes óleos essenciais, no qual $P$. graveolens, que também é conhecido popularmente como gerânio ou malva-cheirosa, possui um forte efeito terapêutico em sua composição. Quanto aos componentes químicos, os ácidos graxos: ácido linolênico, ácido linoléico e ácido palmítico são constituintes majoritários dos óleos de M. Sylvestris. Enquanto P. graveolens é composto principalmente de citronelol, geraniol, formiato de citronelila e mentona. As principais atividades relatadas foram: antimicrobiana, antifúngica, inseticida e antioxidante.

Palavras-chave: Malva sylvestris. Óleos essenciais. Pelargonium graveolens.

\begin{abstract}
Certain medicinal plants have popular names similar to each other. Malva sylvestris and Pelargonium graveolens are species by the same popular name "Malva", and therefore commonly confused, in addition to being used for similar medicinal purposes. The objective of the work was to perform a literature review of the last ten years on the development of scientific knowledge of essential oils of $M$. sylvestris and $P$. graveolens, with an important to identify the main chemical components and biological activities. The results describe the commercial production of these essential essentials, where $P$. graveolens, which is also popularly known as geranium or mallow has a strong therapeutic effect in its composition. As for chemical components, fatty acids: linolenic acid, linoleic acid and palmitic acid are major constituents of $M$. Sylvestris oils. While P. graveolens is composed mainly of citronelol, geraniol, citronellate formate and menthol. The main activities reported were antimicrobial, antifungal, insecticide and antioxidant.
\end{abstract}

Keywords: Essencial oils. Malva sylvestris. Pelargonium graveolens. 


\section{INTRODUÇÃO}

Os produtos naturais podem ser isolados na forma de óleos essenciais, também conhecidos como essencias, óleos voláteis, oleorresinas, resinoides ou óleos etéreos (SERRANO; FIGUEIREDO, 2018). Os óleos essenciais são líquidos hidrofóbicos voláteis extraídos de plantas, na maioria das vezes ricos em aromas. Eles são uma mistura de metabólitos secundários, geralmente terpenoides, que desempenham um papel importante no sistema de defesa da planta (MISHRA et al., 2020).

Os óleos essenciais são identificados com a vantagem de serem seguros e não tóxicos. A Federal Drug Administration (FDA) os enquadra na categoria 'GRAS' (Geralmente Reconhecidos como Seguros) e seus componentes são bem conhecidos por uma variedade de atividades, incluindo antimicrobiana, antiviral, antifúngica e antibacteriana (KULKARNI et al., 2020).

Plantas medicinais costumam receber nomenclatura regional ou popular, e isto, muitas vezes, provoca equívoco tanto na dispensação quanto na administração (MARTINS, 2015). No Brasil, diversas plantas são conhecidas como "malva" e são amplamente utilizadas no tratamento de doenças. Dentre essas plantas, tem-se a Malva sylvestris (Malvaceae), conhecida como Malva-comum (MARTINS, 2017), e Pelargonium graveolens (Geraniaceae) que, apesar de ser de outra família, é conhecida no Brasil como Gerânio ou Malva-cheirosa (GELALETI, 2019).

$M$. sylvestris e $P$. graveolens são espécies de uso popular e os seus óleos essenciais são bastante aproveitados nas indústrias farmacêuticas, agrícolas e cosméticos. São frequentemente confundidas e, em estudos etnobotânicos, ambas apresentam atividade anti-inflamatória, entretanto, atuam especificamente em tecidos diferentes (MARTINS, 2015). Romitelli e Martins (2013) justificam a confusão entre essas espécies devido às semelhanças na morfologia foliar e em seus estudos detectam diferenças anatômicas, auxiliando na taxonomia e classificação dessas espécies.

A $M$. Sylvestris vem sendo utilizada em tratamentos quimioterápicos, anti-inflamatórios e em diversos tipos de infecções, principalmente, em áreas de mucosas como a mucosa vaginal e bucal (CRUZ et al., 2018), além de ser citada para dor no estômago (NEDOPETALSKI; KRUPEK, 2020). Enquanto $P$. graveolens apresenta propriedades e indicações terapêuticas, como antienvelhecimento e relaxante, de combate a acne, antisséptica, adstringente e ação antifúngica (GELALETI, 2019).

Com isso, este trabalho tem como objetivo relatar os principais constituintes químicos e atividades biológicas dos óleos essenciais das espécies $M$. sylvestris e $P$. graveolens, bem como analisar a relação entre estes, dada a importância dessas informações para evitar confusão na medicina popular e na pesquisa de novos produtos com potencial biotecnológico.

\section{METODOLOGIA}

O presente trabalho é uma revisão bibliográfica atualizada. A pesquisa foi realizada por livros, capítulos de livro, dissertações, teses e artigos conduzida nas bases de dados Scielo, PubMed, Science Direct e Google Scholar, em idioma português e inglês, com as seguintes palavras-chave: "Malva" "Malva sylvestris", "Pelargonium graveolens", "Óleos essenciais" / "Essential oils". Foram incluídas publicações entre o período de janeiro de 2010 a julho de 2020. A análise de dados foi realizada em três etapas:

Na primeira etapa, os títulos dos artigos encontrados foram lidos, sendo selecionados aqueles que apresentassem termos relacionados com as espécies e obtenção de óleos essenciais.

$\mathrm{Na}$ segunda etapa, as publicações foram analisadas pela leitura dos Abstracts, selecionando os que mencionassem algum estudo sobre o potencial bioativo e análise dos componentes químicos dos óleos essenciais das espécies de interesse.

$\mathrm{Na}$ terceira etapa, os artigos (Tabelas 1 e 2) foram lidos integralmente e avaliados, a fim de eleger os que comprovassem a atividade biológica do óleo essencial, bem como sua constituição química, sendo utilizado a abordagem de aspectos qualitativos. 


\section{DESENVOLVIMENTO}

\section{Malva sylvestris (Malvaceae)}

Malva sylvestris (Figura 1) é conhecida popularmente como Malva, malva-comum, malvasilvestre, malva-verde, malva-selvagem, pertence à família Malvaceae (BARROS et al., 2010; BOHNEBERGER; MACHADO; DEBIASE, 2019). Pode ser encontrada no continente europeu, africano e americano. No Brasil, não foram encontrados relatos sobre a distribuição dessa espécie de Malva. Possui monografia na Farmacopeia Brasileira, sendo uma das plantas de interesse pelo Sistema Único de Saúde (SUS) e seu consumo aprovado pela Agência Nacional de Vigilância Sanitária (ANVISA) (BRASIL, 2015).

Figura 1 - Malva (Malva sylvestris L., Malvaceae): detalhes da flor e folha, aspecto geral do ramo florido
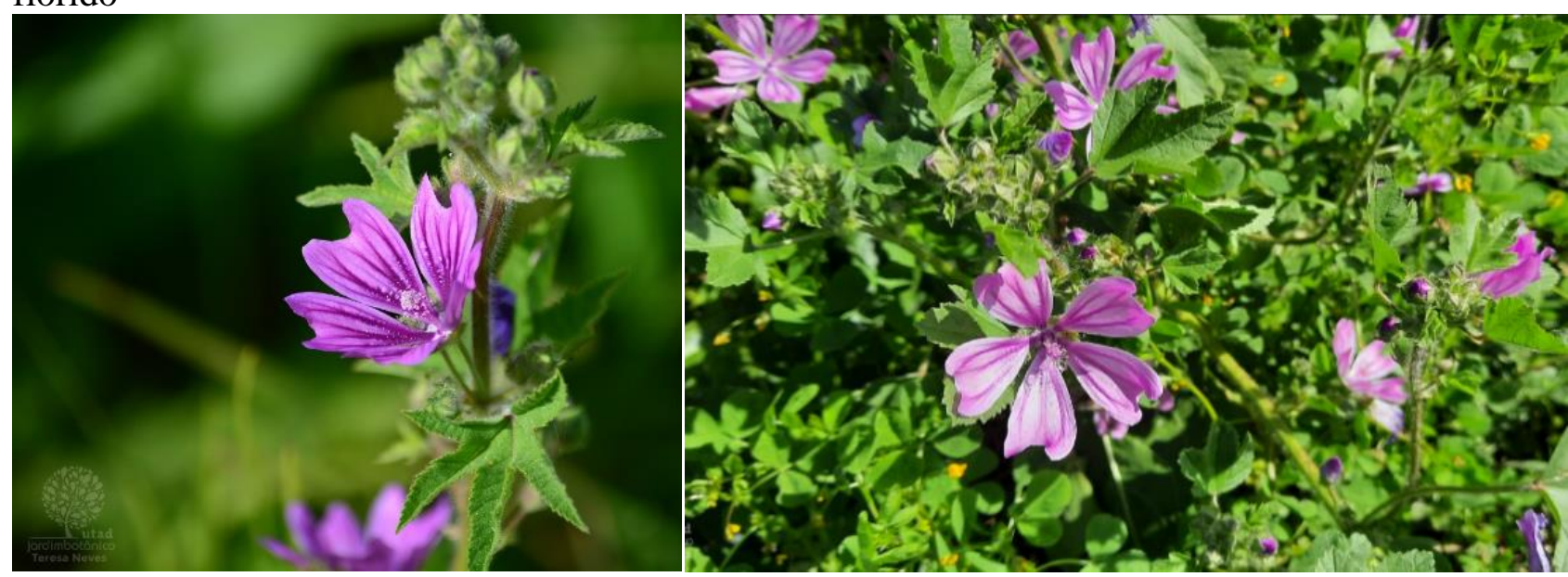

Fonte: UTAD (2015).

É uma espécie de hábito herbáceo, caracteriza-se por apresentar entre 40 e $70 \mathrm{~cm}$ de altura, caule com casca fibrosa, pilosa e ramificada, folhas simples de 7 a $15 \mathrm{~cm}$ de comprimento, com nervação palmada e revestidas de tricomas ásperos. A época de floração ocorre entre a primavera e o verão e as flores atingem de dois a quatro centímetros de diâmetro, com coloração purpúrea ou tons de róseo (DAMEDA; FREITAS; REMPEL, 2014).

Suas folhas, flores e raízes são utilizadas para fins farmacológicos (RAZAVI et al., 2011; ALELUIA et al., 2014). Especialmente, suas flores e folhas são comestíveis, e usadas em saladas e sopas (SPONCHIADO, 2015). De acordo com Barros et al. (2010), as folhas de malva são utilizadas maceradas ou mastigadas, também na forma de infusão, vapor, xarope ou pomadas.

São encontrados relatos de $M$. sylvestris usados na medicina popular do Brasil e outros países para o tratamento da colite e estomatite, nos casos de bronquite crônica, contra furúnculo, queimaduras, dor de dente, contusões, hemorróidas, bem como outros processos inflamatórios (ALRUBAYE; KAIZAL; HAMEED, 2017). Tais atividades farmacológicas e biológicas podem ser atribuídas à presença de antocianidinas, naftaquinonas, flavonóides ou polissacarídeos mucilaginosos que estão em grandes quantidades nos frutos, flores, folhas e raízes da planta (RAZAVI et al., 2011).

\section{Malva sylvestris (Malvaceae) - Constituintes Químicos dos Óleos Essenciais}

Os óleos essenciais, descritos nessa pesquisa (Tabela 1), apresentam diferentes métodos de extração. No que se refere às análises de composições químicas, realiza-se cromatografia gasosa acoplada à espectrometria de massa (GC/MS) como principal técnica. 
Tabela 1 - Características dos óleos essenciais da espécie Malva Sylvestris

\begin{tabular}{|c|c|c|c|c|c|}
\hline $\begin{array}{l}\text { Parte } \\
\text { utilizada }\end{array}$ & $\begin{array}{ll}\text { Método } \\
\text { extração }\end{array}$ & $\begin{array}{l}\text { Componentes } \\
\text { Majoritários }\end{array}$ & $\begin{array}{c}\text { Atividade } \\
\text { biológica do } \\
\text { óleo } \\
\end{array}$ & $\begin{array}{l}\text { Origem da } \\
\text { planta }\end{array}$ & Referência \\
\hline Sementes & $\begin{array}{l}\text { Extração } \\
\text { por solvente } \\
\text { (Soxhlet) }\end{array}$ & $\begin{array}{l}\text { Ácido linoléico: } \\
\text { (49.906\%) } \\
\text { Ácido palmítico: } \\
(22.115 \%) \\
\text { Ácido oleico: } \\
(15.273 \%)\end{array}$ & Antimicrobiana & $\begin{array}{l}\text { Tremecém - } \\
\text { Argélia }\end{array}$ & $\begin{array}{l}\text { Sabri, Belarbi } \\
\text { e Sabri (2013) }\end{array}$ \\
\hline $\begin{array}{l}\text { Folhas e } \\
\text { hastes }\end{array}$ & $\begin{array}{l}\text { Extração } \\
\text { por solvente } \\
\text { (Soxhlet) }\end{array}$ & $\begin{array}{c}\text { Folhas: } \\
\text { Ácido linoléico: } \\
(43,07-50.15 \%) \\
\text { Hastes: } \\
\text { Ácido palmítico: } \\
(22.95-22.97 \%)\end{array}$ & Antioxidante & $\begin{array}{c}\text { Ilam, } \\
\text { Dehloran e } \\
\text { Dezful - Irã }\end{array}$ & $\begin{array}{c}\text { Tabaraki, } \\
\text { Yosefi e } \\
\text { Gharneh } \\
(2012)\end{array}$ \\
\hline Flores & Hidrodestilação & $\begin{array}{c}\text { Hexadecanóico: } \\
(10,1 \%) \\
\text { Pentacosano: }(4,8 \%) \\
\text { 6,10,14-trimetil-2- } \\
\text { pentadecanona: } \\
(4,1 \%)\end{array}$ & --- & Osaka -Japão & $\begin{array}{l}\text { Usami et al., } \\
\text { (2013) }\end{array}$ \\
\hline $\begin{array}{l}\text { Folhas e } \\
\text { Flores }\end{array}$ & $\begin{array}{l}\text { Destilação por } \\
\text { arraste a vapor }\end{array}$ & $\begin{array}{c}\text { Folhas: } \\
\text { Eugenol: }(46,7 \%) \\
\text { Fitol: }(34,4 \%) \\
\text { Flores: } \\
\text { 3-metil tricosano: } \\
\text { 14,9\% }\end{array}$ & Antimicrobiana & $\begin{array}{c}\text { Artern - } \\
\text { Alemanha }\end{array}$ & $\begin{array}{l}\text { Cecotti et al. } \\
\quad(2016)\end{array}$ \\
\hline $\begin{array}{l}\text { Partes } \\
\text { aéreas }\end{array}$ & Hidrodestilação & $\begin{array}{c}\text { 4-Vinil guaiacol: } \\
(19,2 \%) \\
\text { Eugenol: }(14,9 \%) \\
\text { Heptacosano: } \\
(15,1 \%) \\
\text { Ácido linolênico: } \\
(35,5 \%) \\
\text { Ácido palmítico: } \\
(25,6 \%)\end{array}$ & $\begin{array}{l}\text { Atividade anti- } \\
\text { inflamatória e } \\
\text { antimicrobiana }\end{array}$ & $\begin{array}{l}\text { Molise - } \\
\text { Italia }\end{array}$ & $\begin{array}{l}\text { Delfine } \text { et al. } \\
\quad \text { (2017) }\end{array}$ \\
\hline
\end{tabular}

Fonte: a autora.

A composição química de óleos essenciais de M. sylvestris é conhecida por apresentar percentual significativo de ácidos graxos e hidrocarbonetos (DELFINE et al., 2017). Frequentemente são encontrados: ácido hexadecanóico (USAMI et al., 2013), ácido linolênico, ácido linoléico e ácido palmítico (BARROS et al., 2010; LOIZZO et al., 2016).

Sharifi-Rad et al. (2019) realizaram um levantamento bibliográfico sobre a análise fitoquímica de $M$. sylvestris e seus achados mostraram alguns trabalhos avaliando a composição dos óleos essenciais. Foram descritas a presença de malvone A, uma naftoquinona, outros monoterpenos conhecidos, compostos aromáticos, e diterpenos acíclicos tetrahidroxilados, além da presença de polissacarídeos, cumarinas, flavonóides, malvidina 3- (6" -malonilglucosídeo) -5 - glicosídeo, malvalina, escopoletina, polifenóis, niacina, ácido fólico, vitaminas A, C e E, e taninos. Também são relatados terpenóides, como sesquiterpenos, diterpenos e monoterpenos. 
Sabri, Belarbi e Sabri (2013) observaram significativa atividade antimicrobiana dos óleos essenciais das sementes de M. sylvestris, frente às cepas de Enterococcus feacalis e Listeria monocytogenes. Os principais constituintes foram: ácido linoleico (49,906\%), ácido palmítico $(22,115 \%)$, ácido oleico $(15,273 \%)$. O rendimento obtido foi de $9 \%$, pelo método de extração por Soxhlet. Em outro trabalho, realizado por Tabaraki, Yosefi e Gharneh (2012), também utilizando o método de extração Soxhlet, foi comprovado que a capacidade antioxidante das folhas e hastes de $M$. sylvestris é comparável ao espinafre. Os ácidos graxos predominantes foram o ácido linolênico, linoléico, palmítico e oléico, representando $82 \%$ do total de ácidos graxos.

Usami et al. (2013) investigaram a composição do aroma na flor de $M$. sylvestris, obtido por hidrodestilação e analisado por cromatografia gasosa acoplada a espectrometria de massa (GC-MS), cromatografia gasosa acoplada à olfatometria $(\mathrm{GC}-\mathrm{O})$ e análise das diluições do extrato de aroma (AEDA), obtendo-se um rendimento de 0,039\%. Seus compostos majoritários foram: ácido hexadecanóico $(10,1 \%)$, pentacosano $(4,8 \%)$ e 6,10,14-trimetil-2-pentadecanona $(4,1 \%)$. O componente descrito como responsável pelo aroma da planta é o $\beta$-damascenona.

Cecotti et al. (2016) investigaram flores e folhas de $M$. sylvestris através de destilação a vapor, os rendimentos foram de $0,019 \%$ para folhas e $0,012 \%$ para flores. O eugenol foi o composto mais abundante nas folhas, com 46,7\% do óleo total, seguido pelo fitol com 34,4\%. As flores mostraram uma predominância de hidrocarbonetos, entre eles o 3-metil tricosano. Os autores ainda comprovaram maior atividade antimicrobiana dos óleos essenciais, quando comparados com o composto isolado, eugenol. Isso pode ser provocado por um provável efeito sinérgico entre o eugenol e os outros constituintes do óleo, contribuindo para aumentar a atividade biológica contra Paenibacillus larvae e Melisococcus Plutonius, patógenos de abelhas (Apis mellifera).

Delfine et al. (2017) estudaram M. sylvestris em diferentes condições ambientais, cultivadas em dezesseis locais no centro-sul da Itália (Molise), demostrando que o desempenho da cultura foi influenciado pela altitude e, principalmente, pela fertilidade do solo e abastecimento de água. Os autores obtiveram diferentes rendimentos $(0.20 \pm 0.021-0.05 \pm 0.002 \%)$ de extração via hidrodestilação para amostras das partes aéreas da planta. Os óleos essenciais apresentaram boa atividade anti-inflamatória e antimicrobiana. Todas as amostras apresentaram compostos fenólicos e ácidos graxos em suas composições, entretanto as porcentagens em cada ambiente foram diferentes, as quais em alta altitude e baixas temperaturas apresentou maior quantidade de hidrocarbonetos $(51,3 \%)$, e em locais com altas temperaturas, maior quantidade de fenóis $(34,1 \%)$. Os principais componentes foram: 4-Vinil guaiacol (19,2\%), Eugenol (14,9\%), Heptacosano: (15,1\%), Ácido linolênico: (35,5\%), Ácido palmítico: $(25,6 \%)$.

\section{Pelargonium graveolens (Geraniaceae)}

O Pelargonium graveolens L. (Geraniaceae) (Figura 2), popularmente conhecido como gerânio ou malva-cheirosa, é uma espécie aromática nativa da África do Sul (RUSSOMANNO et al., 2013; HAMIDPOUR et al., 2017). O aroma doce e quente, semelhante ao de pétalas de rosas possui alto valor comercial, sendo conhecido como óleo de gerânio, é utilizado como terapêutico (aromaterapia), nas indústrias de cosméticos e perfumaria (ARRIGONI-BLANK et al., 2011; HABER, CLEMENTE, 2013). Atualmente, a China é o maior produtor do óleo essencial de $P$. graveolens, outros países como Índia, Egito, Marrocos e Algéria também representam boa parte da produção mundial (SANDASI et al., 2011).

É uma planta herbácea perene com até 1,3 m de altura e com caule cilíndrico. As folhas são largamente pecioladas, contendo de 5 a 4 lóbulos, arredondados ou cordado-ovados com bordas dentadas (PRADEEPA; KALIDAS; GEETHA, 2016). Possui caules peludos quando jovens, tornando-se lenhosos com a idade. As folhas são verde-claras, espinhosas, esculpidas, aveludadas e com aroma fortemente cítrico. As flores são pequenas, geralmente de coloração rosa (SZUTT; DOŁHAŃCZUK-ŚRÓDKA, 2018). 
Figura 2 - Pelargonium graveolens L. (Geraniaceae): detalhes da flor e folha, aspecto geral do ramo florido
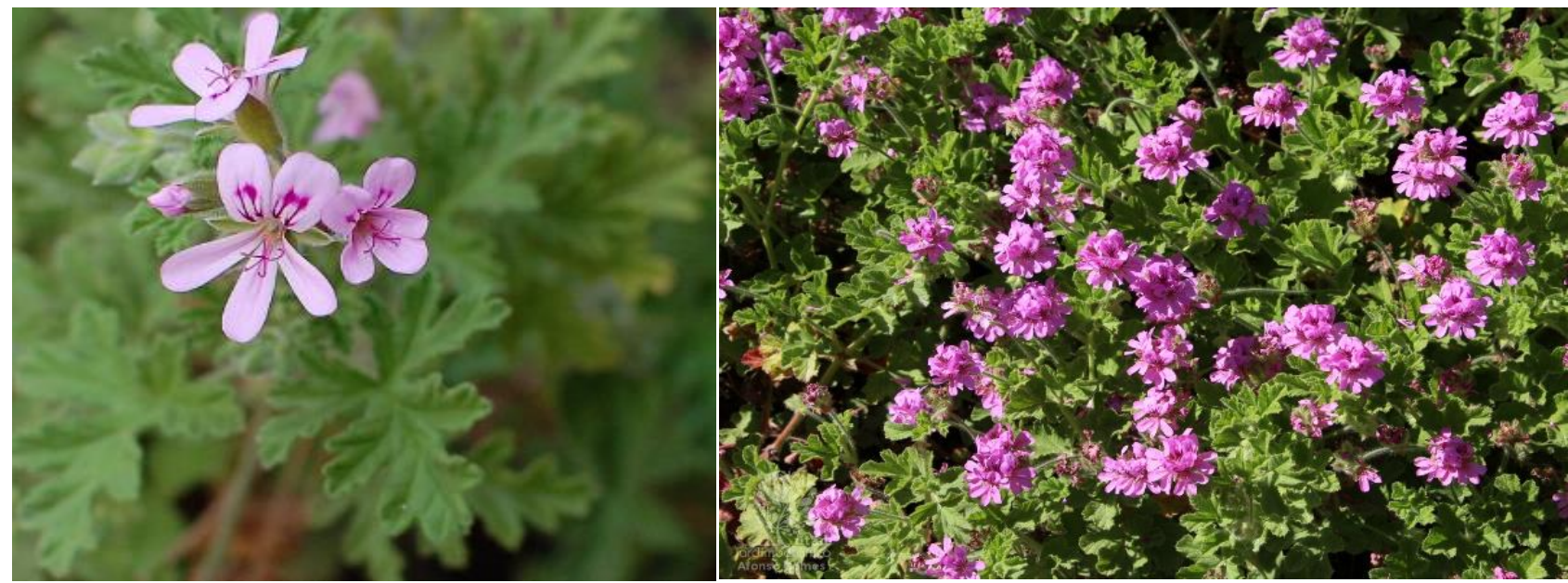

Fonte: UTAD (2015)

Sabe-se que $P$. graveolens é uma planta rica em óleo essencial, e é um dos 20 principais óleos essenciais do mundo (NILOFER et al., 2018). Pesquisas sobre moléculas ativas desses óleos têm comprovado boa atividade antifúngica, anti-inflamatória, antioxidante e efeito antimicrobiano (NEAGU et al., 2018; SADIKI et al., 2019), sendo utilizado como expectorante, afecções das vias respiratórias e para combater problemas de ansiedade e da menopausa (HABER, CLEMENTE, 2013).

Cientistas descobriram significativa redução dos níveis de glicose, por meio da administração oral do óleo essencial de P. graveolens, exercendo, portanto, efeito hipoglicêmico (BOUKHRIS et al., 2012). Džamić et al. (2014) determinaram os constituites do óleo essencial de gerânio comercial e avaliaram a sua atividade antifúngica e antioxidante. Observaram que o óleo essencial foi eficaz contra cepas de Candida albicans e cinco cepas de Aspergillus. Através de uma comparação, concluíram que neste trabalho a atividade antifúngica se apresentou de forma mais acentuada que a antioxidante. Em trabalhos recentes, foi mostrado que o óleo essencial de $P$. graveolens pode reduzir consideravelmente a sensação de medo e nervosismo no momento do parto (FAKARI et al., 2015).

\section{Pelargonium graveolens (Geraniaceae) - Constituintes Químicos dos Óleos Essenciais}

Mesmo sendo de uso comum, ainda há poucos estudos nas áreas da medicina que têm investigado os resultados terapêuticos inerentes ao uso do óleo essencial de Pelargonium graveolens para as diversas condições da saúde, bem como da associação desses resultados aos constituintes químicos do óleo (MONTIBELER et al., 2018).

A diversidade dos compostos químicos abrange flavonoides, ácidos fenólicos, taninos e cumarinas (SARASWATHI et al., 2011; ASGARPANAH; RAMEZANLOO, 2015). Os principais componentes presentes no óleo essencial (Tabela 2) dessa espécie são citronelol, geraniol, linalol, formiato de citronelila, mentona, iso-mentona e nerol (SZUTT; DOŁHAŃCZUK-ŚRÓDKA; SPOREK, 2019).

Niculau et al. (2013) concluíram que os óleos essenciais de P. graveolens apresentam atividade inseticida sobre larvas de terceiro instar de Spodoptera frugiperda. Na sua composição, são encontrados geraniol $(23,1 \%)$, linalol $(11,2 \%)$ e citral $(2,0 \%)$, como componentes majoritários. Com base nos dados, constatou-se que a alta toxicidade do óleo essencial pode estar associada à presença de altos percentuais de geraniol, que causou 30\% de mortalidade das larvas.

Boukhatem, Kameli e Saidi (2013) avaliaram a atividade antimicrobiana do óleo essencial de $P$. graveolens que apresentou bom efeito inibidor contra cepas de Candida, o que sugeriu seu uso em alimentos, como conservantes naturais frente a fungos alimentícios. Os principais constituintes foram citronelol $(30,2 \%)$, formato de citronelil $(9,3 \%)$ e geraniol $(7,6 \%)$. 
Tabela 2 - Características dos óleos essenciais da espécie Pelargonium graveolens

\begin{tabular}{|c|c|c|c|c|c|}
\hline $\begin{array}{l}\text { Parte } \\
\text { utilizada }\end{array}$ & $\begin{array}{ll}\text { Método } \\
\text { extração }\end{array}$ & $\begin{array}{l}\text { Componentes } \\
\text { Majoritários }\end{array}$ & $\begin{array}{c}\text { Atividade biológica } \\
\text { do óleo }\end{array}$ & Origem & Referências \\
\hline Folhas & Hidrodestilação & $\begin{array}{c}\text { Geraniol: }(23,1 \%) \\
\text { Linalol: }(11,2 \%) \\
\text { Citral: }(2,0 \%)\end{array}$ & Inseticida & $\begin{array}{c}\text { São } \\
\text { Cristóvão } \\
\text { - Brasil }\end{array}$ & $\begin{array}{l}\text { Niculau et al. } \\
\text { (2013) }\end{array}$ \\
\hline $\begin{array}{l}\text { Partes } \\
\text { aéreas }\end{array}$ & Hidrodestilação & $\begin{array}{c}\text { Citronelol: }(30,2 \%) \\
\text { Formato de citronelil: } \\
(9,3 \%) \\
\text { Geraniol }(7,6 \%)\end{array}$ & Antimicrobiano & $\begin{array}{l}\text { Blida - } \\
\text { Argélia }\end{array}$ & $\begin{array}{l}\text { Boukhatem, } \\
\text { Kameli e } \\
\text { Saidi (2013) }\end{array}$ \\
\hline Folhas & $\begin{array}{l}\text { Microextração } \\
\text { em fase sólida } \\
(\text { SPME }) \text { e } \\
\text { hidrodestilação }\end{array}$ & $\begin{array}{c}\text { Por SPME: } \\
\text { Citronelol: }(8,15 \%) \\
\text { Formato de citronelil: } \\
(15,41 \%) \\
\text { Mentona: }(15,21 \%) \\
\\
\text { Por hidrodestilação: } \\
\text { Citronelol: }(24,45 \%) \\
\text { Formato de Citronelil: } \\
(13,57 \%) \\
\text { Mentona: }(12,95 \%)\end{array}$ & $\begin{array}{l}\text { Hipoglicêmica } e \\
\text { hipolipidêmica }\end{array}$ & $\begin{array}{c}\text { Amã - } \\
\text { Jordânia }\end{array}$ & $\begin{array}{l}\text { Afifi } e t \text { al. } \\
\text { (2013) }\end{array}$ \\
\hline $\begin{array}{l}\text { Folhas e } \\
\text { Caule }\end{array}$ & Hidrodestilação & $\begin{array}{l}\text { Geraniol: }(50,2 \%) \\
\text { Citronelol: }(14,2 \%)\end{array}$ & Antimicrobiana & $\begin{array}{l}\text { Sarajevo - } \\
\text { Bósnia }\end{array}$ & $\begin{array}{l}\text { Carmem, } \\
\text { Hancu } \\
(2014)\end{array}$ \\
\hline Flores & Hidrodestilação & $\begin{array}{c}\text { Citronelol: }(33,49 \%) \\
\text { Geraniol: }(15,08 \%) \\
\text { Formato de citronelil: } \\
(7,10 \%) \\
\text { Iso-mentona: }(5,1 \%) \\
\text { Linalol: }(4,69 \%) \\
\text { 10-epi- } \gamma \text {-eudesmol: } \\
(3,97 \%) \\
\text { Mentona: }(3,1 \%) \\
\text { Formato de geranil: } \\
(2,88 \%)\end{array}$ & Potencial anestésico & $\begin{array}{c}\text { u-ka } \\
\text { Defne } \\
\text { Essencia - } \\
\text { Turquia }\end{array}$ & $\begin{array}{l}\text { Can et al. } \\
\text { (2018) }\end{array}$ \\
\hline $\begin{array}{l}\text { Partes } \\
\text { aéreas }\end{array}$ & Hidrodestilação & $\begin{array}{c}\text { Citronelol: }(25,24 \%) \\
\text { Geraniol: }(23,36 \%), \\
\text { Formato de citronelil: } \\
(8,35 \%) \\
\text { Linalol: }(7,11 \%) \\
\beta \text {-eudesmol: }(6,13 \%) \\
\text { Formato de geranil: } \\
(4,26 \%) \text { e } \\
\text { Iso- mentona: }(3,37 \%)\end{array}$ & Antifúngica & $\begin{array}{l}\text { Agadir - } \\
\text { Marrocos }\end{array}$ & $\begin{array}{l}\text { Moutaouafiq } \\
\text { et al. (2019) }\end{array}$ \\
\hline
\end{tabular}

Fonte: a autora.

No estudo realizado por Afifi et al. (2013), observou-se atividades hipoglicêmicas e hipolipidêmicas do óleo essencial de $P$. graveolens, extraído de folhas frescas e folhas secas. As técnicas utilizadas foram de microextração em fase sólida (SPME) e hidrodestilação. Os autores relatam que a técnica SPME foi bastante satisfatória ao ser comparada com a técnica tradicional de hidrodestilação, uma vez que é mais rápida, simples e não necessita de solvente. Os resultados obtidos demonstraram que houve diferenças quantitativas e qualitativas em relação à composição dos óleos obtidos por SPME e do óleo hidrodestilado. Entretanto, para ambos os métodos, os principais constituintes identificados foram monoterpenos oxigenados, sendo $69,60 \%$ por hidrodestilação e $50,17 \%$ por SPME. 
Carmem e Hancu (2014) observaram expressiva atividade antioxidante de P. graveolens, conferindo benefícios da planta à saúde. Os óleos essenciais das folhas e do caule foram obtidos por hidrodestilação. Pela análise por CG-EM, oitenta e quatro constituintes do óleo essencial foram identificados, dos quais o geraniol (50,2\%) e citronelol (14,2\%) são os majoritários.

Em estudos inéditos, Can et al. (2018) avaliaram o potencial anestésico do óleo essencial de $P$. graveolens, fornecido por uma empresa comercial. Os resultados foram satisfatórios para sedação e anestesia em peixes das espécies Sciaenochromis fryeri e Labidochromis caeruleus, sugerindo o óleo de gerânio como novo anestésico para a aquicultura. Os principais componentes do óleo foram: citronelol $(33,49 \%)$, geraniol $(15,08 \%)$, formato de citronelil $(7,10 \%)$, iso-mentona $(5,1 \%)$, linalol $(4,69 \%), 10$-epi- $\gamma$-eudesmol $(3,97 \%)$, mentona $(3,1 \%)$ e formato de geranil $(2,88 \%)$.

Moutaouafiq et al. (2019) a fim de estudar plantas aromáticas e medicinais de Marrocos, avaliaram o efeito antifúngico do óleo essencial de $P$. graveolens e suas frações contra quatro fungos responsáveis pela decomposição da madeira (Coniophora puteana, Coriolus versicolor, Poria placenta e Gloeophyllum trabeum). O óleo essencial inibiu o desenvolvimento de todos os fungos testados, sugerindo sua utilização para diminuição do ataque de fungos apodrecedores de madeira. Os principais constituintes identificados foram o Citronelol $(25,24 \%)$, geraniol $(23,36 \%)$, formato de citronelil (8,35\%), linalol (7,11\%), $\beta$-eudesmol $(6,13 \%)$, formato de geranil $(4,26 \%)$ e iso-mentona $(3,37 \%)$.

\section{CONCLUSÃO}

Este trabalho descreveu de forma breve, o estado dos últimos dez anos dos conhecimentos sobre os óleos essenciais de Malva Sylvestris e Pelargonium graveolens. Com a análise dos artigos, conclui-se que ambas as espécies apresentam propriedades bioativas, e, especialmente $P$. graveolens, um forte efeito terapêutico em sua composição. No entanto, somente M. Sylvestris apresenta interesse ao SUS, tratando-se de uma planta medicinal referenciada pelo Ministério da Saúde e pela Anvisa, sendo citada pela presença de mucilagens e uso odontológico.

Apesar de não serem da mesma família, essas plantas são popularmente conhecidas por "Malva" e utilizadas para fins medicinais parecidos. Considerando que os óleos essenciais possuem grande diversidade química, o mecanismo de ação responsável por tais efeitos devem ser compreendidos, uma vez que apresentam bons resultados no tratamento de doenças, e se tornado de interesse sobre o potencial farmacológico.

O óleo essencial de $P$. graveolens (gerânio) é bastante popular, e o número de trabalhos que investigam suas propriedades é consideravelmente maior do que $M$. Sylvestris, mesmo que esta também apresente produção comercial. Os métodos de extração podem variar, entretanto, a hidrodestilação aparece como uma técnica comum. Quanto aos componentes químicos, os ácidos graxos: ácido linolênico, ácido linoléico e ácido palmítico são constituintes majoritários dos óleos de M. Sylvestris. Enquanto P. graveolens é composto principalmente de citronelol, geraniol, formiato de citronelila e mentona. As principais atividades relatadas foram: antimicrobiana, antifúngica, inseticida e antioxidante.

Com isso, vale ressaltar que em grande parte dos trabalhos consultados para realização desta revisão, a composição química dos óleos essenciais ou a bioatividade não foram investigadas, indicando que não há muitos estudos completos sobre estas espécies.

\section{REFERÊNCIAS}

AFIFI, F. et al. Chemical composition and in vitro studies of the essential oil and aqueous extract of Pelargonium graveolens Growing in Jordan for hypoglycaemic and hypolipidemic properties. European Journal of Medicinal Plants, v. 4, n. 2, p. 220-233, 2013. 
ALELUIA, C. M. et al. Fitoterápicos na odontologia. Revista de Odontologia da Universidade de São Paulo, v. 27, n. 2, p. 126-134, 2015.

AL-RUBAYE, A. F.; KAIZAL, A. F.; HAMEED, I. H. Phytochemical screening of methanolic leaves extract of Malva sylvestris. International Journal of Pharmacognosy and Phytochemical Research, v. 9, n. 4, p. 537-552, 2017.

ARRIGONI-BLANK, M. F. et al. Micropropagação e aclimatização de gerânio (Pelargonium graveolens L.). Revista Brasileira de Plantas Medicinais, v. 13, n. 3, p. 271-275, 2011.

ASGARPANAH, J.; RAMEZANLOO, F. An overview on phytopharmacology of Pelargonium graveolens L. Indian Journal of Traditional Knowledge, v. 14, n. 4, p. 558-563, 2015.

BARROS, L.; CARVALHO, A. M.; FERREIRA, I. C. F. R. Leaves, flowers, immature fruits and leafy flowered stems of Malva sylvestris: A comparative study of the nutraceutical potential and composition. Food and Chemical Toxicology, v. 48, n. 6, p. 1466-1472, 2010.

BOHNEBERGER, G.; MACHADO, M. A.; DEBIASE, M. M. Fitoterápicos na odontologia, quando podemos utilizá-los? Brazilian Journal of Health Review, v. 2, n. 4, p. 3504-3517, 2019.

BOUKHATEM, M. N.; KAMELI, A.; SAIDI, F. Essential oil of lgerian rose-scented geranium (Pelargonium graveolens): Chemical composition and antimicrobial activity against food spoilage pathogens. Food Control, v. 34, n. 1, p. 208-213, 2013.

BOUKHRIS, M. et al. Hypoglycemic and antioxidant effects of leaf essential oil of Pelargonium graveolens L'Hér. in alloxan induced diabetic rats. Lipids in Health and Disease, v. 11, n. 81, p. 1$10,2012$.

BRASIL. Ministério da Saúde. Malva sylvestris L. (malva) Organização: Ministério da Saúde e Anvisa. Fonte do Recurso: Ação 20K5 (DAF/ SCTIE/ MS)/2013. Brasília, 2015.

CAN, E. et al. Anesthetic potential of geranium (Pelargonium graveolens) oil for two cichlid species, sciaenochromis fryeri and labidochromis caeruleus. Aquaculture, v. 491, p. 59-64, 2018.

CARMEM, G.; HANCU, G. Antimicrobial and antifungal activity of Pelargonium roseum essential oils. Advanced Pharmaceutical Bulletin, v. 4, n. 2, p. 511-514, 2014.

CECOTTI, R. et al. Chemical characterization of the volatiles of leaves and flowers from cultivated Malva sylvestris var. mauritiana and their antimicrobial activity against the aetiological agents of the european and american foulbrood of honeybees (Apis mellifera). Natural Product Communications, v. 11, n. 10, p. 1527-1530, 2016.

CRUZ, J. H. A. et al. Malva Sylvestris, Vitis Vinífera e Punica Granatum: uma revisão sobre a contribuição para o tratamento de periodontite. Archives of Health Investigation, v. 7, n. 11, p. 486491, 2018.

DAMEDA, M.; FREITAS, E. M.; REMPEL, C. Influência de diferentes substratos e do ácido indolbutírico na propagação vegetativa de Malva Sylvestris L. Caderno Pedagógico, v. 11, n. 1, p. 42-52, 2014. 
DELFINE, S. et al. Variation of Malva sylvestris essential oil yield, chemical composition and biological activity in response to different environments across Southern Italy. Industrial Crops and Products, v. 98, p. 29-37, 2017.

DŽAMIĆ, A. M. et al. Chemical composition, antifungal and antioxidant activity of Pelargonium graveolens essential oil. Journal of Applied Pharmaceutical Science, v. 4, n. 3, p. 1-5, 2014.

FAKARI F. R. et al. Effect of inhalation of aroma of geranium essence on anxiety and physiological parameters during first stage of labor in nulliparous women: a randomized clinical trial. Journal of Caring Sciences, v. 4, n. 2, p. 135-141, 2015.

GELALETI, D. et al. Estabilidade de formulação dermocosmética contendo extrato de Pelargonium Graveolens. Brazilian Journal of Natural Sciences, v. 2, n. 1, p. 16-25, 2019.

HABER, L. L. et al. Características morfológicas, químicas e uso de plantas aromáticas e condimentares In: Haber, L. L.; Clemente, F. M. V. T. Plantas aromáticas e codimentares: uso aplicado na horticultura, p. 19-78, 2013.

HAMIDPOUR, R. et al. Pelargonium graveolens (Rose Geranium) - A novel therapeutic agent for antibacterial, antioxidant, antifungal and diabetics. Archives in Cancer Research, v. 5, n. 134, p. 1$5,2017$.

KULKARNI, S. et al. Computational evaluation of major components from plant essential oils as potent inhibitors of SARS-CoV-2 spike protein. Journal of Molecular Structure, v. 1221, 128823, 2020.

LOIZZO, M. R. et al. Edible flowers: a rich source of phytochemicals with antioxidant and hypoglycemic properties. Journal of Agricultural Food Chemistry, v. 64, n. 12, p. 2467-2474, 2016.

MARTINS, C. A. F. Atividade anti-inflamatória de espécies de "malvas" e análise multivariada para o controle de qualidade de amostras comerciais. 2015. 218f. Tese (Doutorado em Ciências da Saúde) - Universidade Federal do Paraná, Curitiba, 2015.

MARTINS, C. et al. Anti-Inflammatory effect of Malva sylvestris, Sida cordifolia, and Pelargonium graveolens is related to inhibition of prostanoid production. Molecules, v. 22, n. 11, p. 1883, 2017.

MISHRA, A. P. et al. Combination of essential oils in dairy products: A review of their functions and potential benefits. LWT - Food Science and Technology, v. 133, n. 2, e110116, 2020.

MONTIBELER, J. et al. Efetividade da massagem com aromaterapia no estresse da equipe de enfermagem do centro cirúrgico: estudo-piloto. Revista da Escola de Enfermagem da USP, v. 52, n. 03348, 2018.

MOUTAOUAFIQ, S. et al. Antifungal activity of Pelargonium graveolens essential oil and its fractions against wood decay fungi. Journal of Essential Oil Bearing Plants, v. 22, n. 4, p. 11041114, 2019.

NEAGU, A. F. et al. Obtaining and characterization of a selective Pelargonium Graveolens L'hér. dry extract with potential therapeutic activity in metabolic diseases. Farmacia, v. 66, n. 4, p. 592596, 2018. 
NEDOPETALSKI, P. F.; KRUPEK, R. A. O uso de plantas medicinais pela população de União da Vitória-PR: o saber Popular confrontado pelo conhecimento Científico. Arquivos do Mudi, v. 24, n. 1, p. 50-67, 2020.

NICULAU, E. S. et al. Atividade inseticida de óleos essenciais de Pelargonium graveolens l'Herit e Lippia alba (Mill) N. E. Brown sobre Spodoptera frugiperda (J. E. Smith). Química Nova, v. 36, n. 9, p. 1391-1394, 2013.

NILOFER, A. et al. A novel method for survival of rose-scented geranium (Pelargonium graveolens L.) mother plants under extreme climatic conditions. Industrial Crops and Products, v. 126, p. 227237, 2018.

PRADEEPA, M.; KALIDAS, V.; GEETHA, N. Qualitative and quantitative phytochemical analysis and bactericidal activity of Pelargonium graveolens 1'her. International Journal of Applied Pharmaceutics, v. 8, n. 3, p. 7-11, 2016.

RAZAVI, S. M. et al. Bioatividade de Malva sylvestris L., uma planta medicinal do Irã. Iranian Journal of Basic Medical Sciences, v. 14, n. 6, p. 574-579, 2011.

ROMITELLI, I.; MARTINS, M. B. G. Comparison of leaf morphology and anatomy among Malva sylvestris ("gerânioaromático"), Pelargonium graveolens ("falsa-malva") and Pelargonium odoratissimum ("gerânio-de-cheiro"). Revista Brasileira de Plantas Medicinais, v. 15, n. 1, p. 9197, 2013.

RUSSOMANNO, O. M. R. et al. Ocorrência de Cercospora brunkii em gerânio medicinal. Biológico, v. 75, n. 1, p. 23-24, 2013.

SABRI, F. Z.; BELARBI, M.; SABRI, S. Fatty acids profile and antimicrobial activities of the seed oil of Malva sylvestris L. from Algeria. International Journal of Chemical, Environmental and Biological Sciences, v. 1, n. 2, p. 233-235, 2013.

SADIKI, F. Z. et al. Antibacterial properties of the essential oil of Pelargonium Graveolens L'Hér. RHAZES: Green and Applied Chemistry, v. 4, n. 4, p. 17-23, 2019.

SANDASI, M. et al. A quality control method for geranium oil based on vibrational spectroscopy and chemometric data analysis. Vibrational Spectroscopy, v. 57, n. 2, p. 242-247, 2011.

SARASWATHI, J. et al. Phytopharmacological importance of Pelargonium species. Journal of Medicinal Plants Research, v. 5, n. 13, p. 2587-2598, 2011.

SERRANO, C.; FIGUEIREDO, A. C. Óleos essenciais e outros extratos. In: BARATA, A. M. et al. Plantas aromáticas. Instituto Nacional De Investigação Agrária e Veterinária. Portugal: Inocêncio Seita Coelho e Miguel Pestana, p. 11-18, 2018.

SHARIFI-RAD, J. et al. Malva species: Insights on its chemical composition towards pharmacological applications. Phytotherapy Research, v. 34, n. 3, p. 546-567, 2019.

SPONCHIADO, G. Avaliação da eficácia e segurança da Malva sylvestris com potencial atividade para tratamento de disfunções da pele. 2015. 116f. Tese (Doutorado em Ciências da Saúde) - Universidade Federal do Paraná, Curitiba, 2015. 
SZUTT, A.; DOŁHAŃCZUK-ŚRÓDKA, A.; SPOREK, M. Evaluation of chemical composition of essential oils derived from different Pelargonium Species leaves. Ecological Chemistry and Engineering S, v. 26, n. 4, p. 807-816, 2019.

SZUTT. A.; DOŁHAŃCZUK-ŚRÓDKA, A. Influence of abiotic environmental factors on Pelargonium graveolens growth. Proceedings of ECOpole, v. 12, n. 1, p. 87-94, 2018.

TABARAKI, R.; YOSEFI, Z.; GHARNEH, H. A. A. Chemical composition and antioxidant properties of Malva sylvestris L. Journal of Research in Agricultural Science, v. 8, n. 1, p. 59-68, 2012.

USAMI, A. et al. Characterization of aroma-active compounds in dry flower of Malva sylvestris L: by GC-MS-O analysis and OAV calculations. Journal of Oleo Science, v. 62, n. 8, p. 563-570, 2013.

UNIVERSIDADE DE TRÁS-OS-MONTES E ALTO DOURO. Jardim Botânico, Malva sylvestris. Flora Digital de Portugal, Disponível em: https://jb.utad.pt/especie/Malva_sylvestris. Acesso em: 30 de jun de 2021. 